\title{
Exotic Discoveries in Familiar Places: Theory of the Onia and Exotics
}

Richard F. Lebed ${ }^{* \dagger}$

Arizona State University

E-mail: richard. lebedeasu.edu

This brief overview presents an introduction to the heavy-quark exotic hadron candidates, $X, Y, Z$, $P_{c}$, believed to be tetraquark and pentaquark states. After an abridged account of their discovery, we outline the various theoretical pictures employed to describe their structure, with special focus on the new dynamical diquark picture.

16th International Conference on B-Physics at Frontier Machines 2-6 May 2016

Marseille, France

* Speaker.

${ }^{\dagger}$ Work supported in part by the U.S. National Science Foundation under Grant No. PHY-1403891. 


\section{Introduction}

Textbooks tell us that hadrons occur in two conventional types: $q \bar{q}$ mesons and $q q q$ baryons. Especially thorough texts also mention the possibility of other, exotic color-neutral combinations:

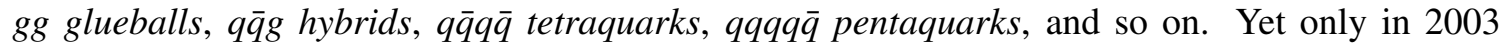
was the first particle clearly identified for which a conventional assignment fails, the $X(3872)$ [1]. Since then, almost 30 additional candidate exotics have been observed, in both the tetraquark and pentaquark sectors. In this brief overview, we examine the major experimental discoveries, the evidence for exoticness, and the variety of theoretical pictures developed to describe their structure.

\section{The Crucial Importance of Quarkonium}

The quark model is now over half a century old. Why did identifying exotics take so long, and why did they first appear in the charmonium $(c \bar{c})$ sector, when so very many light hadron states are known? In part, the answer is that the light sector $(<2.4 \mathrm{GeV})$ is rather crowded. It is easy for exotics made of $u, d, s$ to "hide" amongst ordinary hadrons with the same $J^{P C}$ quantum numbers via mixing. States $\pi_{1}(1400), \pi_{1}(1600)$ with manifestly exotic $J^{P C}=1^{-+}$(hybrid meson candidates) have been observed, but must be extracted from data through a complicated partial-wave analysis. Occasionally, a strong signal for a truly exotic particle can arise, even in multiple experiments, and later disappear [2]: In the early 2000's, evidence for a candidate pentaquark $\Theta^{+}(1540) K-N$ $s$-channel resonance was eventually found to be primarily the result of unfortunate cuts applied to the data, and likely also influenced by kinematical reflections from $t$-channel exchanges. So when the true breakthrough occurred in 2003, it was not instantly accepted by everyone.

The key feature of the $c \bar{c}$ system (and by extension, systems with $b$ quarks) leading to stronger statements about exotics production is that the charm quark is the lightest one substantially heavier than the typical QCD energy scale, $m_{c} \gg \Lambda_{\mathrm{QCD}}$. As was immediately appreciated in the wake of the "November Revolution" of 1974 [3, 4] when the first charmonium state $J / \psi$ was discovered, $m_{c}$ is large enough to allow one to determine the spectrum of charmonium, and the QCD running coupling at this scale, $\alpha_{s}\left(m_{c}\right) \simeq 0.3$, is small enough to justify a simple quantum-mechanical treatment. These facts, even more than the discovery of scaling in deep-inelastic scattering a few years prior, were instrumental in convincing the final skeptics of the reality of quarks and color charges. The most successful treatment in this regard, the so-called "Cornell potential" [5, 6], models the strong interaction between the $c \bar{c}$ pair as

$$
V(r)=-\frac{k \alpha_{s}}{r}+b r
$$

where the first term is the strong Coulomb-like one-gluon exchange, and the second term represents the string or color flux tube-like confining interaction. Various small modifications (e.g., spinspin or spin-orbit terms) can be included, but once the particular form of $V(r)$ is decided, it can be fed directly back into the Schrödinger equation to obtain the explicit $c \bar{c}$ spectrum and wave functions. Such models have successfully predicted the entire $c \bar{c}$ spectrum below the lowest opencharm $\left(D^{0} \bar{D}^{0}\right)$ threshold and also a number of states above it (black lines in Fig. 1), and similarly in the $b \bar{b}$ system. Possessing explicit wave functions also allows one to predict radiative and hadronic decay rates, which generally agree well with data. 
The existence of many additional states in the region of neutral $c \bar{c}$ states (red lines in Fig. 1) thus strongly points to a large and complex system of charmoniumlike exotics. That they truly contain a $c \bar{c}$ valence pair is evidenced by the fact that every observed decay channel contains a $c \bar{c}$ pair. Unless we have somehow grossly misunderstood conventional $c \bar{c}$ systems, these extra states must certainly be QCD exotics. Moreover, as several charged charmoniumlike states (discussed below) have been discovered, the neutral $c \bar{c}$ or $c \bar{c} g$ structures are insufficient to describe them all.

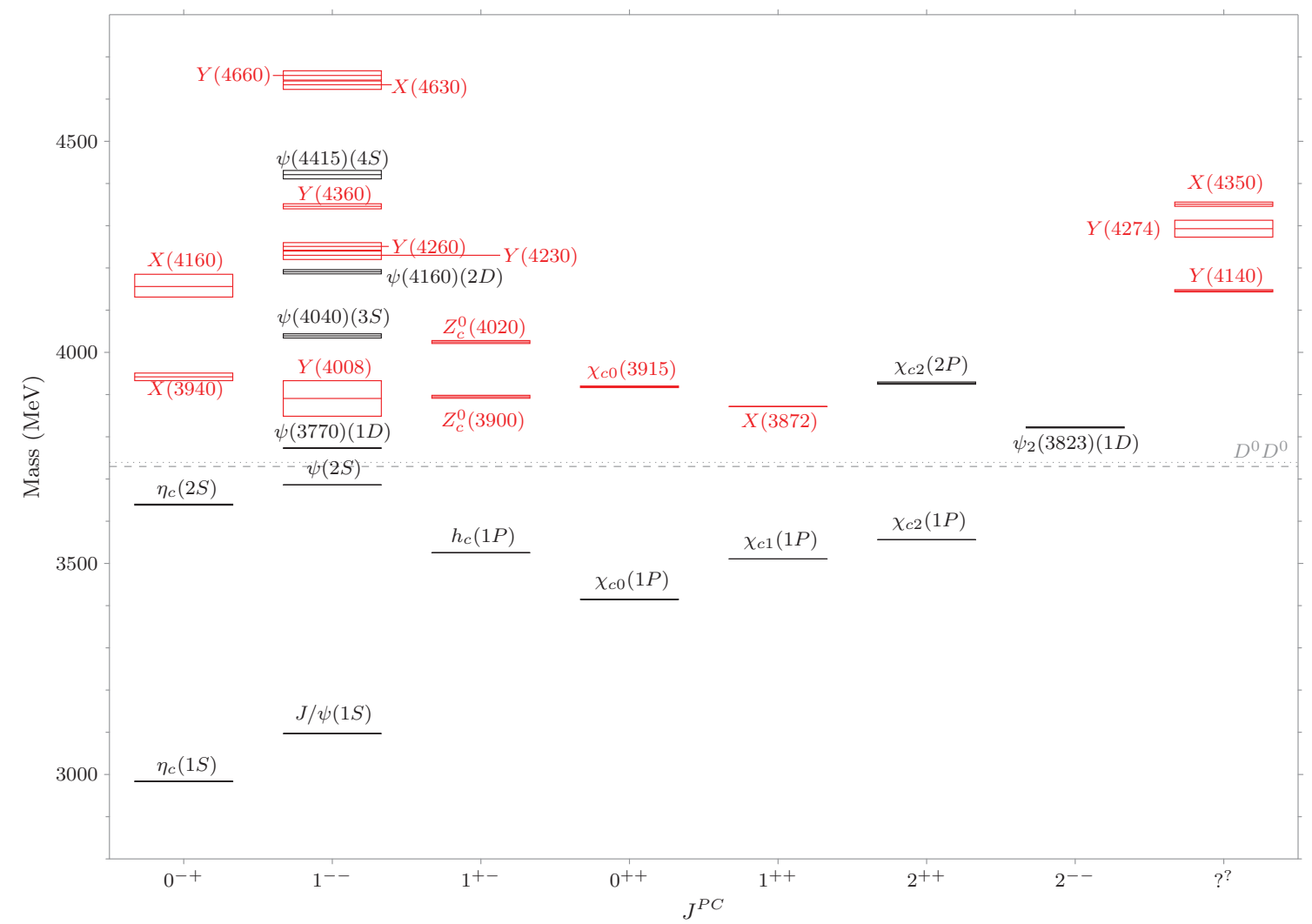

Figure 1: The neutral charmonium system. Black lines indicate the conventional $c \bar{c}$ states predicted by quark-potential models that have been experimentally observed. Red lines indicate the charmoniumlike exotic states. Rectangles give the $1 \sigma$ uncertainty of measured mass values. The lowest open-charm $\left(D^{0} \bar{D}^{0}\right)$ threshold is indicated as a dashed line.

\section{Discovery of the Exotic Hadrons $X, Y, Z, P_{c}$}

The $X(3872)$ was discovered by Belle in 2003 as a resonance of $\pi^{+} \pi^{-} J / \psi$ in the decay $B \rightarrow K\left(\pi^{+} \pi^{-} J / \psi\right)$. It is worth recalling that the principal physics goal of Belle was not to look for exotics but to find $C P$ violation in the neutral $B$ sector; very likely, many more such surprise discoveries lie in store for the $b$-physics community. The existence of $X(3872)$ has since been confirmed by multiple experiments. Its quantum numbers are $J^{P C}=1^{++}$, but the nearest conventional $c \bar{c}$ state, the yet-undiscovered $\chi_{c 1}(2 P)$, is predicted to lie many 10 's of $\mathrm{MeV}$ higher. Its measured 
mass is $m_{X(3872)}=3871.69 \pm 0.17 \mathrm{MeV}$, astonishingly close to the $D^{0} \bar{D}^{* 0}$ threshold:

$$
m_{X(3872)}-m_{D^{* 0}}-m_{D^{0}}=-0.11 \pm 0.21 \mathrm{MeV},
$$

fueling endless speculation (first proposed in [7]) that it is a $D^{0} \bar{D}^{* 0}$ molecule. In any case, it is believed to be a $c \bar{c} u \bar{u}$ state. Its width is remarkably small, $\Gamma_{X(3872)}<1.2 \mathrm{MeV}$, incompatible with being an excited conventional $c \bar{c}$ state. The label " $X$ " emphasizes that its true structure and place in the family of hadrons still remains unknown.

In 2005, the BaBar Collaboration discovered the second charmoniumlike exotic, $Y$ (4260) [8]. They exploited the initial-state radiation (ISR) technique, in which the beam $e^{ \pm}$pair emits a real photon before colliding; then the state emerging from the collision vertex carries the $J^{P C}=1^{--}$of the virtual photon. $Y(4260)$ has been confirmed by other experiments, and is charmoniumlike, as evidenced both by its mass and decay modes, such as $\pi^{+} \pi^{-} J / \psi$. It is believed exotic because all of the expected conventional $c \bar{c}$ states in the $1^{--}$channel up to the $4 S \psi(4415)$ have been seen (see Fig. 1). Subsequently, several other exotics have been observed in the $1^{--}$channel, all labeled $Y$.

The $Y(4260)$ was used in experiments by both the BESIII [9] and Belle [10] Collaborations for the first confirmation of a charged charmoniumlike state, $Z_{c}^{+}(3900)$, in 2013: It appears as a resonance in the decay $Y(4260) \rightarrow \pi^{-}\left(\pi^{+} J / \psi\right)$, and its quantum numbers have been determined to be $J^{P}=1^{+}$. As a charged state, its minimal flavor content is $c \bar{c} u \bar{d}$, meaning that it was the first manifestly exotic state ever confirmed beyond $5 \sigma$ by two experiments. While news of this discovery should have become much more widely known in the physics community, the cautionary example of the $\Theta^{+}(1535)$ was still a recent memory; what if all of the "states" discovered up to this point turned out not really to be states, but rather brilliant forgeries?

One important feature of a true resonance (in contrast to an unfortunate statistical "bump" in cross-section data) is a characteristic interference of its complex production amplitude $f(s)$ with nonresonant background. In the idealized case of an isolated Breit-Wigner resonance,

$$
f(s)=\frac{\Gamma / 2}{M-\sqrt{s}-i \Gamma / 2},
$$

the scattering phase shift $\delta \equiv \arg f(s)$ increases rapidly from 0 to $\pi$, creating a characteristic looping behavior in the Argand plane. In 2014, the LHCb Collaboration not only confirmed [11] the existence (at 13.9 $\sigma$ ) of a charged charmoniumlike state, $Z^{-}(4430) \rightarrow \pi^{-} \psi^{\prime}$ (first seen by Belle in 2008 [12]) with $J^{P}=1^{+}$, but also measured the phase shift in the resonant region and found it to be compatible with the expected looping behavior. While nature could be especially unkind and generate this behavior even in the absence of a resonance, such an accident seems unlikely, and moreover, further tests by $\mathrm{LHCb}$ [13] support that $Z^{-}$(4430) is not merely a kinematical reflection. As of 2014, one could state with some confidence that we had entered the Age of the Third Hadron.

Indeed, one may also say that the Age of the Fourth Hadron followed only one year later. In

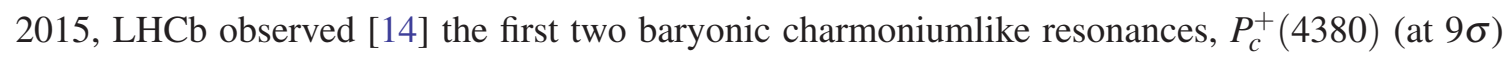
and $P_{c}^{+}$(4450) (at 12 $\sigma$ ) decaying into $J / \psi p$, and therefore possessing pentaquark $c \bar{c} u u d$ quantum numbers. While the $J^{P}$ assignments are not yet completely established, the preferred solution gives opposite parities and one state with spin $3 / 2$ and the other with spin 5/2. Again, rapid phase variation in the vicinity of the peaks, consistent with resonant behavior, was observed [although resembling the ideal Breit-Wigner behavior rather less for the $\left.P_{c}^{+}(4380)\right]$. 
This discussion has been, of necessity, extremely brief and does not mention all the other exotic candidates, the supporting experiments, the conflicting signals, and the dead ends. Nor does it mention that a few exotic candidates have been observed in the $b \bar{b}$ sector as well. An expansive discussion appears in the recent review Ref. [15]. As of the time of the BEAUTY 2016 Conference, 29 exotics had been observed, of which 24 are in the $c \bar{c}$ sector, 4 are in the (much less explored) $b \bar{b}$ sector (See, e.g., [16, 17]), and 1 in the bsud sector [18]. Of these, 12 have been confirmed by a second experiment, and only 1 of the other 17 (the last) has been challenged by strong contrary evidence [19]. Of particular interest to this conference, a large number of $b$-containing exotics almost certainly await discovery.

\section{How Are the Exotics Assembled?}

The existence of tetraquark and pentaquark states having been established experimentally, we briefly explore their structure as composite multiquark particles. On the order of 1000 theoretical papers have appeared since the discovery of the $X(3872)$, so it will be impossible to include any but the earliest references to a particular approach, and for specificity, we focus on the tetraquarks.

Given a $q \bar{q} q \bar{q}$ system, simple SU(3) color algebra shows that color singlets can be formed in exactly two ways, which correspond to the two possible independent $q \bar{q}$ pairings. While it is tempting to describe these pairings as corresponding to the formation of hadronic molecules [e.g., in the case of $X(3872)$, either $D^{0} \bar{D}^{* 0}$ or $\left.J / \psi \omega\right]$, group theory alone does not determine hadronic structure or dynamics. The quarks can segregate themselves spatially in several other ways that do not resemble a molecular configuration. Let us first discuss the popular non-molecular pictures.

Charmoniumlike hybrids were first modeled in Ref. [20]). They may explain some of the neutral exotics, but not the $Z^{ \pm}$. Furthermore, for most values of $J^{P C}$, hybrids are expected from lattice QCD to lie higher in mass than the observed exotics [21].

The hadrocharmonium picture [22] assumes that the heavy $c \bar{c}$ pair in a tetraquark resides at the center of a cloud provided by the lighter two quarks. Such models are best at describing decays of the exotic into conventional charmonium, but are somewhat less successful for open-charm decays.

The diquark picture (sometimes confusingly referred to as "tetraquark") is based on the idea that quarks have a little-appreciated attractive channel: In addition to the well-known color-singlet $q \bar{q}$ combination, a $q q$ pair at short distance exhibits a strong attraction, fully one-half as large, into a color- $\overline{\mathbf{3}}$. One can imagine multiquark hadrons with compact diquark $(q q)$ or antidiquark $(\bar{q} \bar{q})$ components. The spectroscopy of such states has been developed in Refs. [23, 24] and, for example, gives a simple explanation for the $Y$ states as $L=1$ excitations of the ground states such as $X(3872)$. However, diquark models also tend to greatly overpredict the number of states expected.

It is even possible for apparent resonances not to be true states at all, but rather an effect caused by the rapid opening of a nearby two-meson threshold. The complex analyticity properties of the associated two-point function create a effective attraction that can, in certain circumstances, produce a cusp-shaped profile resembling in all respects a resonance. The most well-known of these kinematic effects is the cusp effect first suggested for the heavy exotics in Ref. [25]; however, rescattering effects due to the production of loops of virtual particles can have similar effects. Nevertheless, not every exotic lies especially close to a threshold [the $Z^{-}(4430)$ is a prime example], and states as narrow as the $X(3872)$ are very difficult to explain purely as a kinematical effect. 
We return at last to the simple hadronic molecule picture, first proposed for $c \bar{c}$ systems in [26, 27]. A number of the exotics lie suspiciously close to hadron thresholds [e.g., Eq. (3.1)]. However, not all are, as has just been noted for the $Z^{-}$(4430). Moreover, some $X Y Z$ states lie slightly above a hadronic threshold [e.g., $Y(4260)$ lies about $30 \mathrm{MeV}$ above the $D_{s}^{*} \bar{D}_{s}^{*}$ threshold], creating the awkward problem of a bound state with positive binding energy. In the well-studied case of the $X(3872)$, one would expect a state with such a tiny binding energy - the central value is 20 times smaller than the binding energy of a deuteron, already considered weakly bound - to be especially delicate. The $X(3872)$ might appear in $B$ decays, but one would expect them to be blown apart at the high energies of collider experiments. Plenty of $D^{0}$ and $\bar{D}^{* 0}$ mesons should be produced in prompt production in the central interaction region, but very few should be produced close enough in phase space to coalesce into a molecular configuration, even including strong final-state interaction effects. And yet, CDF [28] and CMS [29] produced X(3872) as copiously as conventional $c \bar{c}$ states. Charmoniumlike hadronic molecules may certainly exist, but the $X(3872)$ does not seem to fit the expected profile.

It is entirely possible that no single structure accommodates all of the exotic states. Some could be molecules, some hybrids, some kinematical effects, etc. Or they could be quantummechanical mixtures of several of these. For example, allowing both molecular and conventional $c \bar{c}$ components for the $X(3872)$ is very helpful in accommodating its currently known properties. Each picture deserves continued study to map out its successes, shortcomings, and limits of applicability.

\section{A New Dynamical Picture for the $X, Y, Z, P_{c}$}

A feature shared by all of the aforementioned pictures is a reliance, either explicitly or implicitly, on essentially static structures (e.g., the formation of recognizable mesonic degrees of freedom, or diquarks in well-defined orbitals). They do not obviously incorporate the full complexity of QCD dynamics in what are, after all, rather short-lived states. This author and collaborators therefore recently developed a new picture [30] to accommodate these facts.

The claim is that at least some of the observed tetraquark states are bound states of diquarkantidiquark pairs, as suggested above. However, unlike other familiar bound states, the components are not in a static molecular configuration. Rather, the diquarks are created with a large relative momentum, and rapidly separate from each other (hence the name dynamical diquark picture). Since the diquarks are not color neutral, they cannot, by confinement, separate arbitrarily far. Their kinetic energy is converted into the potential energy of the color flux tube connecting them, until the pair is brought to rest at some substantial separation, at which point hadronization occurs though the large-distance tails of meson wave functions stretching from the $q$ 's of the diquarks to the $\bar{q}$ 's of the antidiquarks. But these tails are exponentially suppressed, leading to suppressed decay transitions and hence, observably narrow widths. Why should this type of hadronization occur, rather than a simple breaking of the flux tube at this point, creating an additional $q \bar{q}$ and resolving the system via baryon-antibaryon creation? The lowest such threshold is $\Lambda_{c} \bar{\Lambda}_{c}$ at $4573 \mathrm{MeV}$, and indeed, the lowest exotic candidate $[X(4630)]$ above this threshold is observed [31] to decay only into $\Lambda_{c} \bar{\Lambda}_{c}$.

As an example, consider the production of $Z^{+}(4430)$ in nonleptonic $\bar{B}^{0}=b \bar{d}$ decay (production in $b$-factories and colliders is similar). The weak transition $b \rightarrow c s \bar{c}$ suggests the obvious "colorallowed" open-charm decay route $(b \bar{d}) \rightarrow(c \bar{d})(s \bar{c})=D^{(*)+} \bar{D}_{s}^{(*)-}$, where parentheses indicate the 
compact quark combinations. This particular quark transition occurs about $22 \%$ of the time, and the given 2-body decays amount to about $5 \%$ of $\bar{B}^{0}$ decays [32]. In such decays, the c.m. momentum of the hadrons is $\simeq 1700 \mathrm{MeV}$, simply reflecting the large energy release due to $m_{b} \gg m_{c}$.

Of course, several other decay mechanisms are available, including color-suppressed decays, penguins, and so on. In the dynamical diquark picture, in some fraction of decays the formation of a diquark-antidiquark pair is favorable. In the case of $\bar{B}^{0}$, let the weak decay $b \rightarrow \operatorname{cs} \bar{c}$ be accompanied by the creation of an additional $u \bar{u}$ pair from the vacuum. Some fraction of the time, the $(u c)$ and $(\bar{c} \bar{d})$ pairs appear in sufficient proximity to form color-triplet diquarks rather than color-singlet $q \bar{q}$ pairs. The remaining $(s \bar{u})$ forms a $K^{-}$, so the production process, just as is experimentally observed, is $\bar{B}^{0} \rightarrow K^{-} Z^{+}$(4430); were the diquarks color neutral, this process would be a conventional 3body hadronic decay. Instead, the $(u c)$ and $(\bar{c} \bar{d})$ diquarks separate until forced to come to rest and hadronize as described above, into the observed pair $\psi^{\prime} \pi^{+}$; this configuration defines the $Z^{+}(4430)$.

To support this picture, note a remarkable experimental feature of the $Z^{+}(4430)$ : It heavily prefers to decay to the $J^{P C}=1^{--} 2 S$ state $\psi^{\prime}$ rather than the $1 S$ state $J / \psi$ by at least a factor of 10 [33], despite the significantly larger phase space for the latter. This fact is easy to understand if one calculates the final separation $r_{Z}$ of the diquark-antidiquark pair using, say, the Cornell potential; one finds $r_{Z}=1.16 \mathrm{fm}$. In comparison, the same potential gives charge radii of the $c \bar{c}$ states, $\left\langle r_{J / \psi}\right\rangle=0.39 \mathrm{fm}$ and $\left\langle r_{\psi^{\prime}}\right\rangle=0.80 \mathrm{fm}$. A simple matter of greater wave function overlap for the $\psi^{\prime}$ in the dynamical diquark model explains its preferred status in $Z^{+}(4430)$ decay.

Finally, a few words about the $P_{c}^{+}$states: The color attraction for $\mathbf{3} \times \mathbf{3} \rightarrow \overline{\mathbf{3}}$ applies not just to diquarks, but can be compounded successively to triquarks and beyond. In Ref. [34], the discovery channel $\Lambda_{b} \rightarrow P_{c}^{+} K^{-}$is seen to be an exact analogue to the $\bar{B}^{0} \rightarrow Z^{+}(4430) K^{-}$, except that the diquark $(\bar{c} \bar{d})$ is replaced by the color-3 antitriquark $[\bar{c}(u d)]$.

\section{The Present and the Future}

The past two years have provided confirmation of the existence of tetraquarks and observation, at high statistical significance, of the pentaquark, the third and fourth classes of hadron. To date, almost 30 such states $\left(X, Y, Z, P_{c}\right)$ have been observed. Several theoretical pictures have been developed to describe their structure (molecules, hybrids, diquark-antidiquark states, hadrocharmonium, and kinematic effects), and while each one has appealing features, none of them appears to universally explain all of the new states. We have also described the new "dynamical diquark" picture, designed to allow creation of a spatially large hadronic system still strongly bound by color forces.

The field of heavy-quark QCD exotics is nonetheless strongly data-driven. $b$-physics experiments will continue to lead the way in the development of this rich subject (as evidenced by reports in this conference on the latest exotics results from ATLAS, BESIII, CMS, and LHCb [35], and prospects for the BelleII upgrade [36]), and a final understanding of these states will become clear.

\section{References}

[1] S.K. Choi et al. [Belle Collaboration], / psi decays,” Phys. Rev. Lett. 91, 262001 (2003) [hep-ex/0309032].

[2] R.A. Schumacher, AIP Conf. Proc. 842, 409 (2006) [nucl-ex/0512042]. 
[3] J.J. Aubert et al. [E598 Collaboration], Phys. Rev. Lett. 33, 1404 (1974).

[4] J.E. Augustin et al. [SLAC-SP-017 Collaboration], Phys. Rev. Lett. 33, 1406 (1974) [Adv. Exp. Phys. 5, 141 (1976)].

[5] E. Eichten, K. Gottfried, T. Kinoshita, K.D. Lane, and T.-M. Yan, Phys. Rev. D 17, 3090 (1978) Erratum: [Phys. Rev. D 21, 313 (1980)].

[6] E. Eichten, K. Gottfried, T. Kinoshita, K.D. Lane, and T.-M. Yan, Phys. Rev. D 21, 203 (1980).

[7] N.A. Törnqvist, Phys. Lett. B 590, 209 (2004) [hep-ph/0402237].

[8] B. Aubert et al. [BaBar Collaboration], Phys. Rev. Lett. 95, 142001 (2005) [hep-ex/0506081].

[9] M. Ablikim et al. [BESIII Collaboration], Phys. Rev. Lett. 110, 252001 (2013) [arXiv:1303.5949 [hep-ex]].

[10] Z.Q. Liu et al. [Belle Collaboration], Phys. Rev. Lett. 110, 252002 (2013) [arXiv:1304.0121 [hep-ex]].

[11] R. Aaij et al. [LHCb Collaboration], Phys. Rev. Lett. 112, 222002 (2014) [arXiv:1404.1903 [hep-ex]].

[12] S.K. Choi et al. [Belle Collaboration], Phys. Rev. Lett. 100, 142001 (2008) [arXiv:0708.1790 [hep-ex]].

[13] R. Aaij et al. [LHCb Collaboration], Phys. Rev. D 92, 112009 (2015) [arXiv:1510.01951 [hep-ex]].

[14] R. Aaij et al. [LHCb Collaboration], Phys. Rev. Lett. 115, 072001 (2015) [arXiv:1507.03414 [hep-ex]].

[15] H.X. Chen, W. Chen, X. Liu, and S.L. Zhu, arXiv:1601.02092 [hep-ph].

[16] A. Bondar et al. [Belle Collaboration], Phys. Rev. Lett. 108, 122001 (2012) [arXiv:1110.2251 [hep-ex]].

[17] P. Krokovny et al. [Belle Collaboration], Phys. Rev. D 88, no. 5, 052016 (2013) [arXiv:1308.2646 [hep-ex]].

[18] V.M. Abazov et al. [D0 Collaboration], [arXiv:1602.07588 [hep-ex]].

[19] The LHCb Collaboration [LHCb Collaboration], LHCb-CONF-2016-004, CERN-LHCb-CONF-2016-004.

[20] R. Giles and S.H.H. Tye, Phys. Rev. Lett. 37, 1175 (1976).

[21] E. Braaten, C. Langmack, and D.H. Smith, Phys. Rev. D 90, 014044 (2014) [arXiv:1402.0438 [hep-ph]].

[22] M.B. Voloshin, Prog. Part. Nucl. Phys. 61, 455 (2008) [arXiv:0711.4556 [hep-ph]].

[23] L. Maiani, F. Piccinini, A.D. Polosa, and V. Riquer, Phys. Rev. D 71, 014028 (2005) [hep-ph/0412098].

[24] L. Maiani, F. Piccinini, A.D. Polosa, and V. Riquer, Phys. Rev. D 89, 114010 (2014) [arXiv:1405.1551 [hep-ph]].

[25] D.V. Bugg, Phys. Lett. B 598, 8 (2004) [hep-ph/0406293].

[26] M.B. Voloshin and L.B. Okun, JETP Lett. 23, 333 (1976) [Pisma Zh. Eksp. Teor. Fiz. 23, 369 (1976)].

[27] A. De Rújula, H. Georgi, and S.L. Glashow, Phys. Rev. Lett. 38, 317 (1977).

[28] A. Abulencia et al. [CDF Collaboration], Phys. Rev. Lett. 98, 132002 (2007) [hep-ex/0612053]. 
[29] S. Chatrchyan et al. [CMS Collaboration], JHEP 1304, 154 (2013) [arXiv:1302.3968 [hep-ex]].

[30] S.J. Brodsky, D.S. Hwang, and R.F. Lebed, Phys. Rev. Lett. 113, 112001 (2014) [arXiv:1406.7281 [hep-ph]].

[31] G. Pakhlova et al. [Belle Collaboration], Phys. Rev. Lett. 101, 172001 (2008) [arXiv:0807.4458 [hep-ex]].

[32] K.A. Olive et al. [Particle Data Group Collaboration], Chin. Phys. C 38, 090001 (2014).

[33] K. Chilikin et al. [Belle Collaboration], Phys. Rev. D 90, 112009 (2014) [arXiv:1408.6457 [hep-ex]].

[34] R.F. Lebed, Phys. Lett. B 749, 454 (2015) [arXiv:1507.05867 [hep-ph]].

[35] See talks in this volume by V. Lyubushkin, L. Wang, L. Cristella, and D.C. Craik, respectively.

[36] See talks in this volume by Y. Okada and N. Minakashi. 